Three cases with tetraplegia are accompanied by dysfunction of the bladder and the rectum, and by disproportionately more motor impairment of the upper han of the lower extremities. But the fairly good prognosis may be implied in these cases. The lower extremities tend to recover motor power first, bladder unction is improved next, and finally strength in the upper extremities reappears. However, the recovery in the upper extremities is not complete, but remain paralysis in slight degree.

Other three cases with cervical spondylosis complain a radiating neuralgia as a irritation syndrome in the nerve roots of $\mathrm{C}_{5}$ and $\mathrm{C}_{6}$, and this neuralgia becomes more painful in the standing position of the body or by the hyperextension of cervical spine. No paralysis is recognized in the lower extremities of these cases.

According to the observations mentioned above, it is strongly suggested that the impairments of spinal cord and its nerve root might be easely occured by slight extrinsic strength, especially by hyperextension of cervical spine in the cases with cervical spondylosis.

\title{
125. A Case of Traumatic Hematomyelia Caused by Hypertensive Injury of the Cervical Spine
}

\author{
Hajime NAGAI and Masayuki HAYASHI \\ 2nd Department of Surgery, Nagoya University School of Medicine
}

A man aged fifty-two years gave a clear history of injury by forcible hyperextension of the cervical spine. On 3rd day after injury, he was gradually paralysed in both arms and legs and had anesthesia below the segmental level of C5-6.

Radiographs of the spine showed no abnormality.

He died from respiratory paralysis two weeks after the injury.

Necropsy showed diffuse invading intramedullar hemorrhage at vertebral level between Th5 and upper cervical cord.

A case of cervical traumatic hematomyelia was described in which there was no evidence of damage to vertebrae or ligaments.

The reasons why the above mentioned traumatic hematomyelia may occur was discussed. 